\title{
Twelve species of nematodes: new records for India
}

\author{
Padma Bohra
}

Desert Regional Centre, Zoological Survey of India, Jodhpur, Rajasthan, India

Email: bohrapadma@gmail.com

\begin{abstract}
Twelve species of nematodes belonging to orders Tylenchida (6 sp.) and Dorylaimda (6 sp.). Under nine genera of nine familes; were reported as.new record from India.Samples were collected from agriculture fields, surrondigs of lakes of Udaipur and Sitamata Wild Life Sanctuary, Rajasthan.
\end{abstract}

Keywords: Family, genera, nematodes, new records, species, taxonomy.

A review of literature reveals that Rajasthan State does not feature in nematlogical literature till Arya (1957) reported the first plant parasitic nematode (Meloidogyne sp.) from the Thar Desert from Jodhpur exactly after 57 years of the first record of plant nematodes from India in 1901. Khera (1967-1969) and his associates identified and reported a significant number of species, including many new to science, from Jodhpur, Pali, Bikaner and Jaisalmer districts of Rajasthan (Sethi \& Swarup 1968 a, b; Tikyani \& Khera 1968; Tikyani et al. 1969; Nandkumar \& Khera 1969, 1970; Khera 1971; Bajaj \& Jairajpuri 1979; Bohra \& Baqri 2000, 2003; Baqri \& Bohra 2001, 2003; Bohra 2008, 2011).

Despite all these studies, our knowledge of

Date of publication (online): 26 August 2012

Date of publication (print): 26 August 2012

ISSN 0974-7907 (online) | 0974-7893 (print)

Editor: Anonimity requested

Manuscript details:

Ms \# 02703

Received 10 February 2011

Final received 19 April 2012

Finally accepted 05 July 2012

Citation: Bohra, P. (2012). Twelve species of nematodes: new records for India. Journal of Threatened Taxa 4(9): 2889-2899.

Copyright: (๑ Padma Bohra 2012. Creative Commons Attribution 3.0 Unported License. JoTT allows unrestricted use of this article in any medium for non-profit purposes, reproduction and distribution by providing adequate credit to the authors and the source of publication.

Acknowledgements: The Author is grateful to Dr. K. Venkataraman Director, Zoological Survey of India, Kolkata for providing research facilities for preparation of the paper.

\section{OPEN ACGESS | FREE DOWNLOAD}

freshwater nematode fauna from Rajasthan is meagre. Keeping this point in view, attempts were made to collect water samples from different depths of the lakes of Udaipur and from submerged plants surrounding the lakes. However, from the samples taken from different depths of the lakes, the recovery of nematodes was not satisfactory.

The present paper is based on the collection brought from agricultural fields, surroundings of lakes of Udaipur during (2009-2010) surveys, districts of Udaipur, Rajsamand and Sitamata Wildlife Sanctuary, Chittorgarh. The analysis of samples yielded a wide variety of nematodes which included 12 species of nematodes belonging to Orders Tylenchida (6 spp.) and Dorylaimida (6 spp.) as new records for India. Besides, brief descriptions, illustrations and information on host(s) and locality(ies) of these species have also been provided.

\section{Material and methods}

Forty soil samples were collected around the roots of host plants with the help of a shovel from depths of $5-15 \mathrm{~cm}$ in two surveys of six days duration. The soil/ sediment samples were collected in a polythene bag, labelled and brought to the laboratory. Samples can be stored at $6-10^{\circ} \mathrm{C}$ to keep the nematodes physiologically young and active. Lower storage temperature $\left(4-5^{\circ} \mathrm{C}\right)$ may cause chilling injury. Exposer to $40^{\circ} \mathrm{C}$ or above even for a short time kill some species moderate temperature for storage of samples is $10-15{ }^{\circ} \mathrm{C}$. Samples were processed through Cobb's sieving and decantation technique. Nematodes were killed and fixed in hot $4 \%$ formaldehyde and kept for dehydration in desiccators. Body measurements were taken for specific species identification. Presently specimens are deposited in National Zoological Collection of Desert Regional Centre, Jodhpur, Rajasthan.

Abbreviations: $\mathrm{L}=$ body length $(\mathrm{mm} / \mu \mathrm{m})$; $a=$ body length/ maximum body width; $b=$ body length/oesophageal length; $c=$ body length/tail length; $c^{\prime}=$ tail length/body width at anus; $V=d$ 


\section{Results}

The analysis of samples yielded a wide variety of nematodes which included 12 species belonging to nine genera of nine families of Orders Tylenchida and Dorylaimida.

Systematic Account:

Order: Tylenchida Thorne, 1949

Family: Tylenchidae Orley, 1880

\section{Zanechus zanchus Siddiqi, 1979}

(Fig. 1 A-B and Image 1 A-C)

Material examined: Two females, two males; 15.x.2009, coll. P. Kadela; Reg.No. IV/2332

Measurements: Females: $\mathrm{L}=0.70-0.75 \mathrm{~mm}$; a $=39.47-41.21 ; \mathrm{b}=6.8-7.0 ; \mathrm{c}=12.50-14.00 ; \mathrm{c}^{\prime}=$ 3.2-3.5; $\mathrm{V}=80-82$. Males: $\mathrm{L}=0.56-0.57 \mathrm{~mm} ; \mathrm{a}=$ $31.30-33.30 ; \mathrm{b}=5.8-6.2 ; \mathrm{c}=11.34-12.54$;

Description: Female: Body ventrally arcuate upon fixation. Cuticle deeply annulated, annules contiguous. Lip region low, continuous with body contour. Stylet $8-9 \mu \mathrm{m}$ long. Median bulb oval. Vulva post equatorial. Post vulval uterine sac absent. Tail 50-55 $\mu \mathrm{m}$ long, elongate conoid to a hooked tip (Fig. B)

Male: Spicules 15-16 $\mu \mathrm{m}$ long. Gubernaculum trough-shaped, 7-8 $\mu \mathrm{m}$ long; fixed. Cloacal lips pointed forming a narrow tube. Bursa adanal. Tail elongate conoid to hooked tip.

Habitat: Collected from moist soil surrounding the bank of Pichola Lake, Udaipur.

Remarks: The measurement of present specimens fits well within the range given by Siddiqi (1979).

\section{Family: Hoplolaimidae Filipjev,1934}

\section{Helicotylenchus californicus Sher, 1966}

(Fig. 1, C-D and Image 2 A-C)

Material examined: Three females, 05.i.2010, coll. R. Sultana, Reg.No. IV/2350.

Measurements: Females: $\mathrm{L}=0.57-0.61 \mathrm{~mm} ; \mathrm{a}=$ $19-27 ; \mathrm{b}=5.6-6.9 ; \mathrm{c}=30.50-32.50 ; \mathrm{c}^{\prime}=0.8-1.3 ; \mathrm{V}$ $=59-62$.

Description: Female: Body spirally coiled upon fixation. Lip region hemispherical, marked by four annules. Stylet $25-26 \mu \mathrm{m}$ long, basal knobs with flattened anterior surfaces. Excretory pore just posterior to oesophago-intestinal valve. Ovaries paired. Phasmid two annules anterior to anus. Tail with pronounced ventral projection, terminus irregularly hemispherical, marked by eight annules (Fig. C.)

Habitat and locality: Collected from soil around roots of wheat (Triticum aestivum) at village Poonmal (Sayra), Udaipur District, Rajasthan, India.

Remarks: H. californicus can be distinguished from related species $H$. bryophilus in having shorter stylet knobs with flattened stylet; offset anterior surface; spermathecae and irregular ventral projection of the female tail (Stylet 28-32 $\mu \mathrm{m}$; ventral projection of tail hemispherical in H. bryophilus).
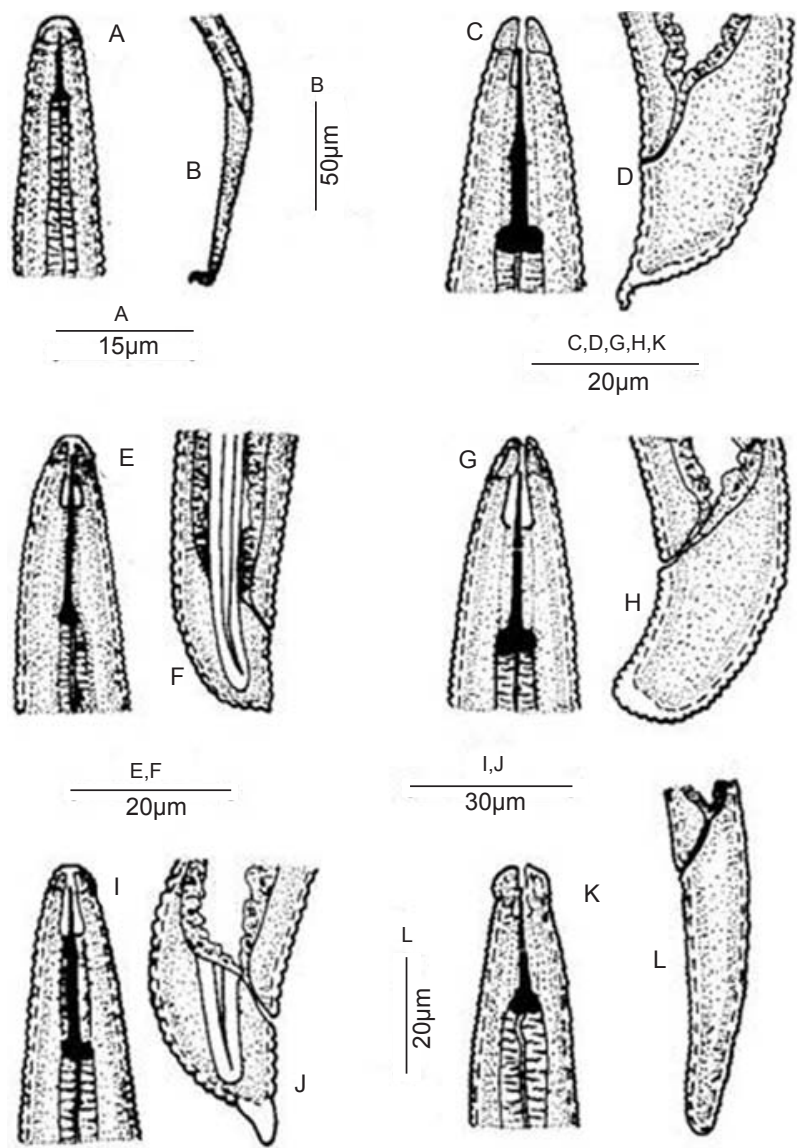

Figure 1. Zanenchus zanchus Siddiqi, 1979

A - Anterior end; B - Posterior end.

Helicotylenchus californicus Sher, 1966

C - Anterior end; D - Posterior end.

Helicotylenchus densibullatus Siddiqi, 1972

E - Anterior end; F - Posterior end.

Helicotylenchus minzi Sher, 1966

$\mathrm{G}$ - Anterior end; H - Posterior end.

Helicotylenchus talonus Siddiqi, 1972

I - Anterior end; J - Posterior end

Neodolichorhynchus judithae (Andrássy, 1962) Jairajpuri \&

Hunt, 1984

K - Anterior end; L - Posterior end. 

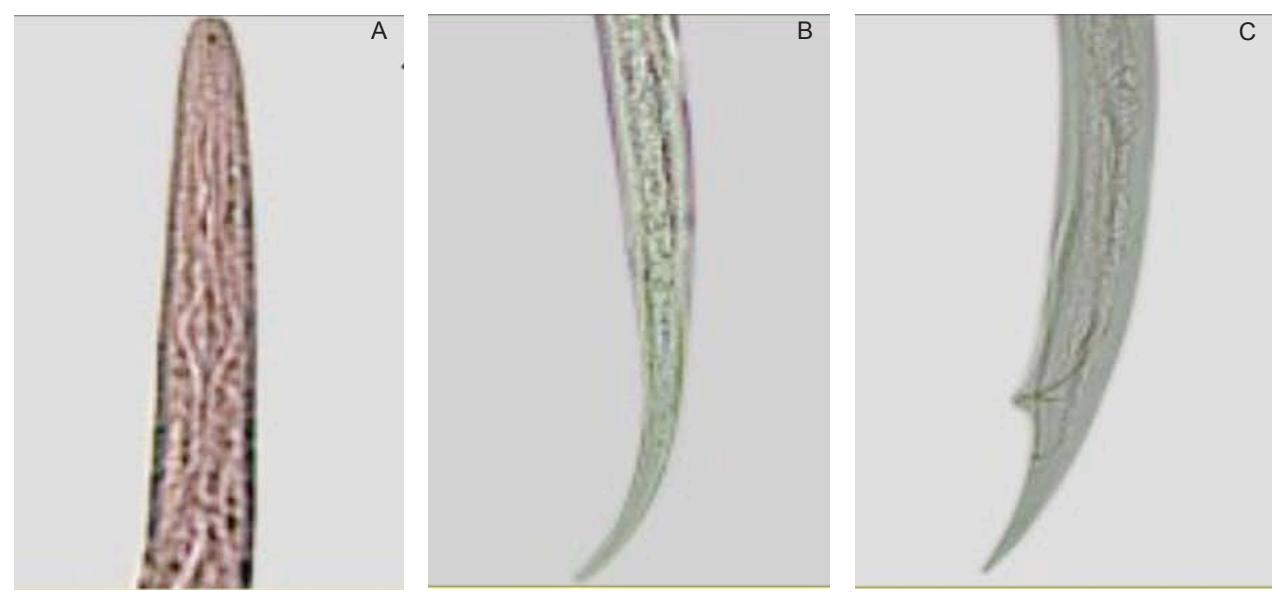

Image I. Zanenchus zanchus Siddiqi, 1979. A - Anterior end; B - Posterior end; C - Male - Posterior end
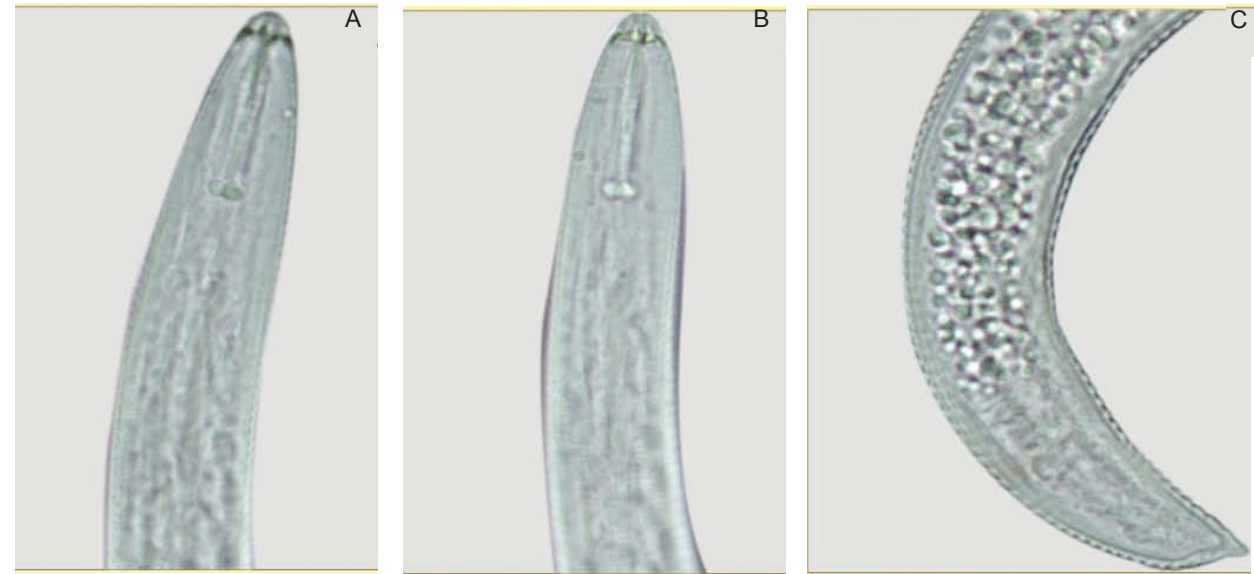

Image 2. Helicotylenchus californicus Sher, 1966. A - Anterior end; B - Basal Knobs; C - Posterior end.
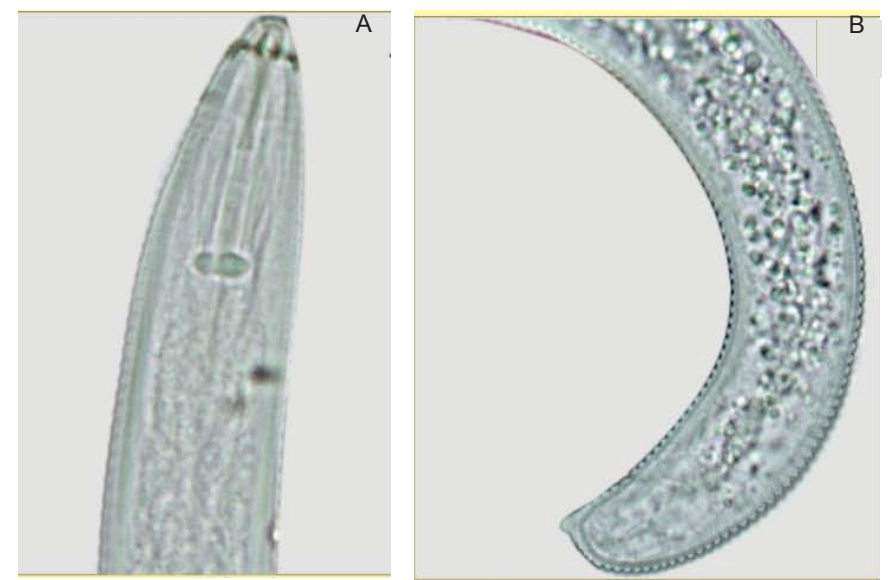

Image 3. Helicotylenchus densibullatus Siddiqi, 1972. A - Anterior end; B - Posterior end 


\section{Helicotylenchus densibullatus Siddiqi, 1972}

(Fig. 1, E-F and Image $3 \mathrm{~A}-\mathrm{B}$ )

Material examined: Five females, 31.xii.2009, coll. P. Bohra, Reg.No. IV/2329.

Measurements: Females: $\mathrm{L}=0.43-0.44 \mathrm{~mm} ; \mathrm{a}=$ $22-24 ; \mathrm{b}=4.7-6.2 ; \mathrm{c}=42-44 ; \mathrm{c}^{\prime}=0.76-0.90 ; \mathrm{V}=$ 65-67.

Description: Female: Body spirally coiled upon fixation. Lip region hemispheroid and marked by three distinct annules. Labial sclerotization moderate. Lateral fields marked by four incisures, outer ones slightly crenate. Stylet 21-23 $\mu \mathrm{m}$ long. Median oesophageal bulb spheroidal in shape. Excretory pore opposite to oesophago-intestinal junction. Ovaries paired. Intestine not exceeding into rectum. Tail dorsally convex-conoid to a short usually acute ventral projection, with 7-10 annules. Annules becoming slightly coarser ventrally towards distal end.

Habitat and locality: Collected from soil around roots of Banana (Musa balbisiana) from Kakarwa district, Rajasamand, Rajasthan, India.

Remarks: H. densibullatus is characterized in having basal knobs of stylet appearing to be amalgamated with the shaft, not distinctly marked off individually (hence specific name); it comes closer to $H$. indicus Siddiqi, 1963 which has narrow truricurate lip region, indistinct labial annules, a more conspicuous labial sclerotization, from $H$. elegans Roman, 1965 has well separated anteriorly cupped stylet knobs. Lip region bearing five or six annules.

\section{Helicotylenchus minzi Sher, 1966}

(Fig. 1, G-H and Image 4 A-B)

Material examined: Three females, 27.7.2009, Coll. P.Bohra, Reg. No. IV/2331 \& 2277.

Measurements: Females: L $=0.61-0.80 \mathrm{~mm}$; a $=26-34 ; \mathrm{b}=5.5-6.4 ; \mathrm{c}=46-71 ; \mathrm{c}^{\prime}=0.5-1.0 ; \mathrm{V}=$ $60-65$.

Description: Female: Body in loose spiral shape. Lip region truncated, marked by 5 annules. Stylet 26$28 \mu \mathrm{m}$ long, basal knobs indented anteriorly. Excretory pore anterior to oesophago-intestinal, junction. Hemizonid at level of excretory pore. Female reproductive system amphidelphic. Spermatheca offset; with sperms. Phasmids 2-4 annules anterior to anus. Tail more curved dorsally, terminus irregularly hemispherical bearing 10-11 annules.

Habitat: Collected from moist soil around the bank of Pichola lake, Udaipur District, Rajasthan, India.

Remarks: $H$. minzi can be distinguished from $H$. canadiensis by having shorter stylet; (stylet $30-33 \mu \mathrm{m}$; female tail terminus hemispherical to irregularly hemispherical with six to 12 annules in $H$. canadiensis).

\section{Helicotylenchus talonus Siddiqi, 1972}

(Fig. 1, I-J and Image 5 A-B)

Material examined: 3 females, 31.xii.2009; coll. P. Bohra, Reg.No. IV/2344.

Measurements: Females: $\mathrm{L}=0.41-0.46 \mathrm{~mm}$; $\mathrm{a}=$ $22-24 ; \mathrm{b}=5.8-6.5 ; \mathrm{c}=1.1-1.2 ; \mathrm{c}^{\prime}=0.8-0.9 ; \mathrm{V}=$ 62-66.

Description: Female: Body spirally curved upon fixation. Lateral fields marked by four incisures. Lip region hemispheroid, distinctly marked by $4-5$ labial annules. Stylet 19-20 $\mu \mathrm{m}$ long; basal knobs large with flattened to concave anterior surface-median oesophageal bulb oval. Ovaries paired. Tail dorsally convex-conoid, with 3-7 annules ventrally and a large, bluntly rounded, claw-like unstriated ventral projection.

Habitat and locality: Collected from soil around roots of Lemon (Citrus reticulate) at Kakarwa, Rajasamand District, Rajasthan, India.

Remarks: H. talonus Siddiqi, 1972 comes close to H. glissus Thorne \& Malek, 1968; H. bradys Thorne \& Malek, 1968 and H. borinquensis Roman, 1965. From H. glissus it differs in having a distinctly annulated lip region, a wider tail projection and inner incisures of the lateral fields usually fusing distally on tail. From $H$. bradys it can be differentiated by spear length (spear 29-33 $\mu$ long in H. bradys), finer body annules ( $3 \mu$ wide at mid-body in $H$. bradys) and fewer tail annules. From $H$. borinquensis it can be separated by its fewer tail annules, a large tail projection and phasmids which are closer to the anal level. 

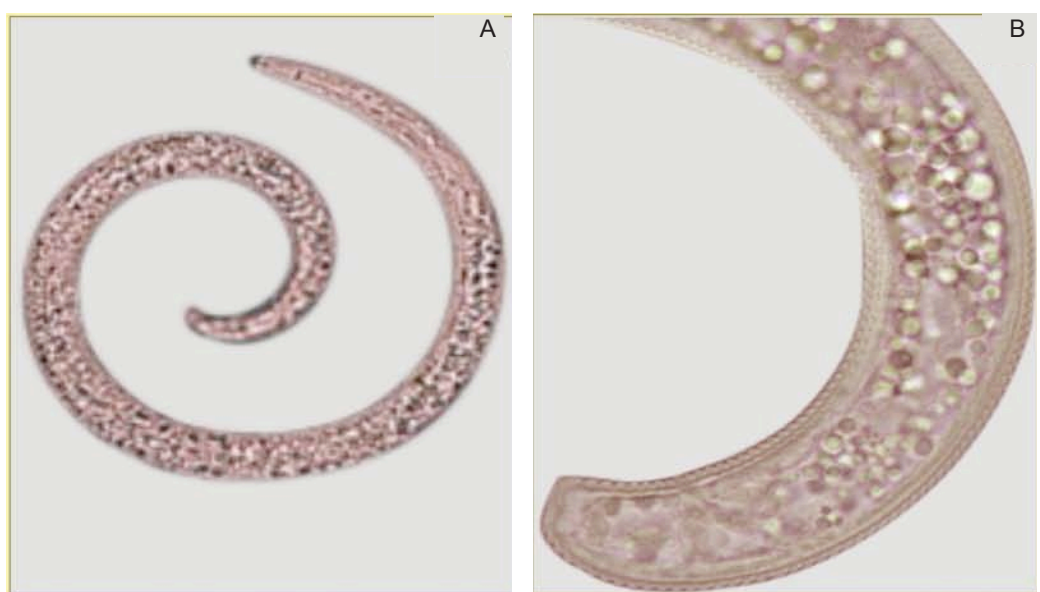

Image 4. Helicotylenchus minzi Sher, 1966. A - Entire Female; B - Posterior end.

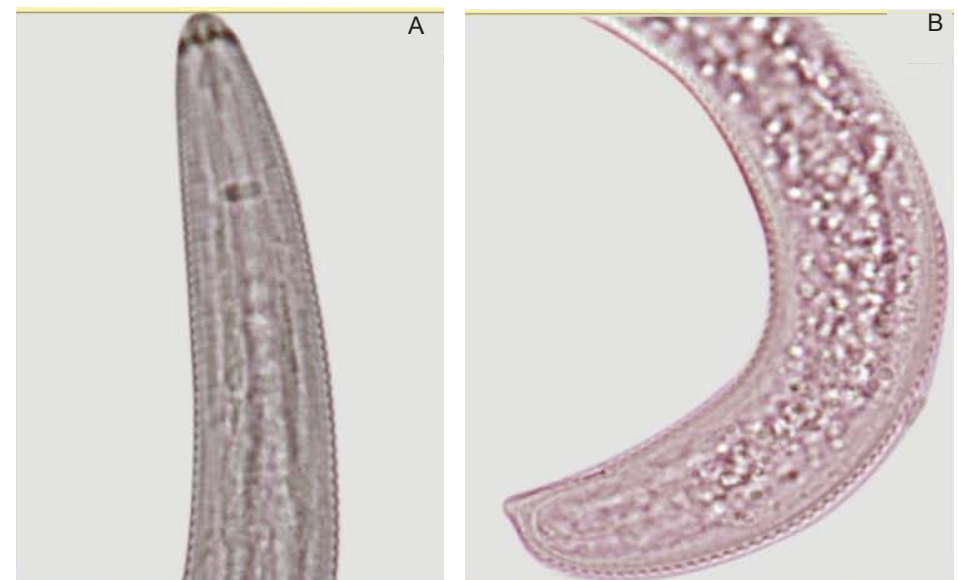

Image 5. Helicotylenchus talonus Siddiqi, 1972; A - Anterior end; B - Posterior end.

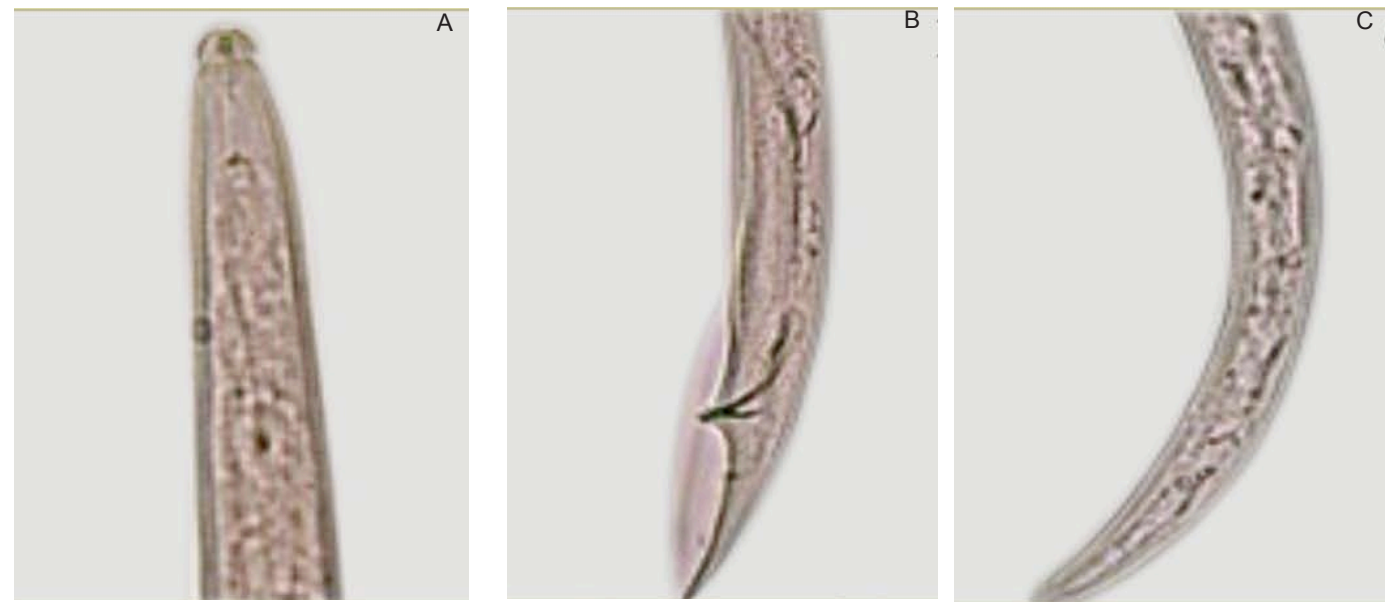

Image 6. Neodolichorhynchus judithae (Andrássy, 1962) Jairajpuri \& Hunt, 1984. A - Anterior end; B - Male Posterior end; C - Female Posterior end. 


\section{Family: Telotylenchidae Siddiqi, 1960}

Neodolichorhynchus (Neodolichorhynchus) judithae (Andrassy, 1962) Jairajpuri \& Hunt, 1984

(Fig. 1, K-L and Image $6 \mathrm{~A}-\mathrm{C}$ )

Material examined: Two females, two males; 14.x.2009, coll. R. Sultana, Reg.No. IV/2336.

Measurements: Females: $\mathrm{L}=0.51-0.55 \mathrm{~mm} ; \mathrm{a}=$ $27-28 ; \mathrm{b}=4.9-5.6 ; \mathrm{c}=17-18 ; \mathrm{c}^{\prime}=2.25-3.55 ; \mathrm{V}=$ 57-58; Males: $\mathrm{L}=0.55-0.60 \mathrm{~mm} ; \mathrm{a}=27 ; \mathrm{b}=4.5-5.3$; $\mathrm{c}=17 ; \mathrm{c}^{\prime}=3.55-4.00$.

Description: Female: Body ventrally arcuate upon fixation. Annules prominent, marked by deep transverse striae. Cuticle with 6-14 longitudinal ridges or lamellae outside lateral fields. Lateral fields with four incisures variably aerolated. Lip region rounded, labial framework moderately sclerotized. Stylet 12 $17 \mu \mathrm{m}$ long; conus solid appearing in anterior third. Median bulb round or oval. Female reproductive system amphidelphic. Tail 27-36 $\mu \mathrm{m}$ long, conoid to subcylindrical, ending in a rounded often lobe-like hyaline terminus.

Male: Spicules 20-22 $\mu \mathrm{m}$ long. Gubernaculum 12-15 $\mathrm{m}$ long, protrusible. Tail 32-36 $\mu \mathrm{m}$ long, enveloped by large bursa, 59-62 $\mu \mathrm{m}$ long; Bursa not notched at tail terminus (Image 6 B)

Habitat and locality: Collected from soil around roots of Chickoo (Manikara zopota) at Nathdwara district, Udaipur, Rajasthan, India.

Remarks: The measurements of specimens are in conformity with those given by Jairajpuri \& Hunt, 1984.

Order: Dorylaimida Pearse, 1942

Family: Dorylaimidae de Man, 1876

\section{Mesodorylaimus subtiloides (Paetzold, 1958) Andrassy, 1959}

(Fig. $2 \mathrm{~A}-\mathrm{B}$ and Image $7 \mathrm{~A}-\mathrm{C}$ )

Material examined: Two females, two males, 14.x.2009, coll. P. Kadela, Reg.No. IV/ 2283.

Measurements: Females: $\mathrm{L}=1.17-1.21 \mathrm{~mm}$; $\mathrm{a}=$ $31-41 ; b=4.45-4.92 ; c=8.36-10.85 ; c^{\prime}=8.5-8.6 ; \mathrm{V}$ =47-50. Males: $\mathrm{L}=0.88-1.06 \mathrm{~mm} ; \mathrm{a}=31-35 ; \mathrm{b}=$ $3.50-3.98 ; \mathrm{c}=40-50 ; \mathrm{T}=52-55$.

Description: Female: Body almost straight in females and ventrally arcuate at posterior extremities in females. Lip region continuous with adjoining body. Odontostyle 12-14 $\mu \mathrm{m}$ long, aperture one third odontostyle length. Guiding ring thin single. Odontophore 18-20 $\mu \mathrm{m}$ long. Oesophagus enlarges in its middle. Expanded part of oesophagus occupies $47-50 \%$ total oesophageal length. Vulva transverse. Ovaries paired. Tail 117-145 $\mu \mathrm{m}$ long, elongate filifom about 8.5-8.6 anal body width long.

Male: Spicules 42-43 $\mu \mathrm{m}$ long. Lateral guiding pieces $12-13 \mu \mathrm{m}$ long. Ventromedian supplements 8-9. Tail short conoid.

Habitat: Collected from moist soil around the bank of water body around Aarmpura Range, Sitamata Wildlife Sanctuary, Chittorgarh.

Remarks: Identification done with the help of
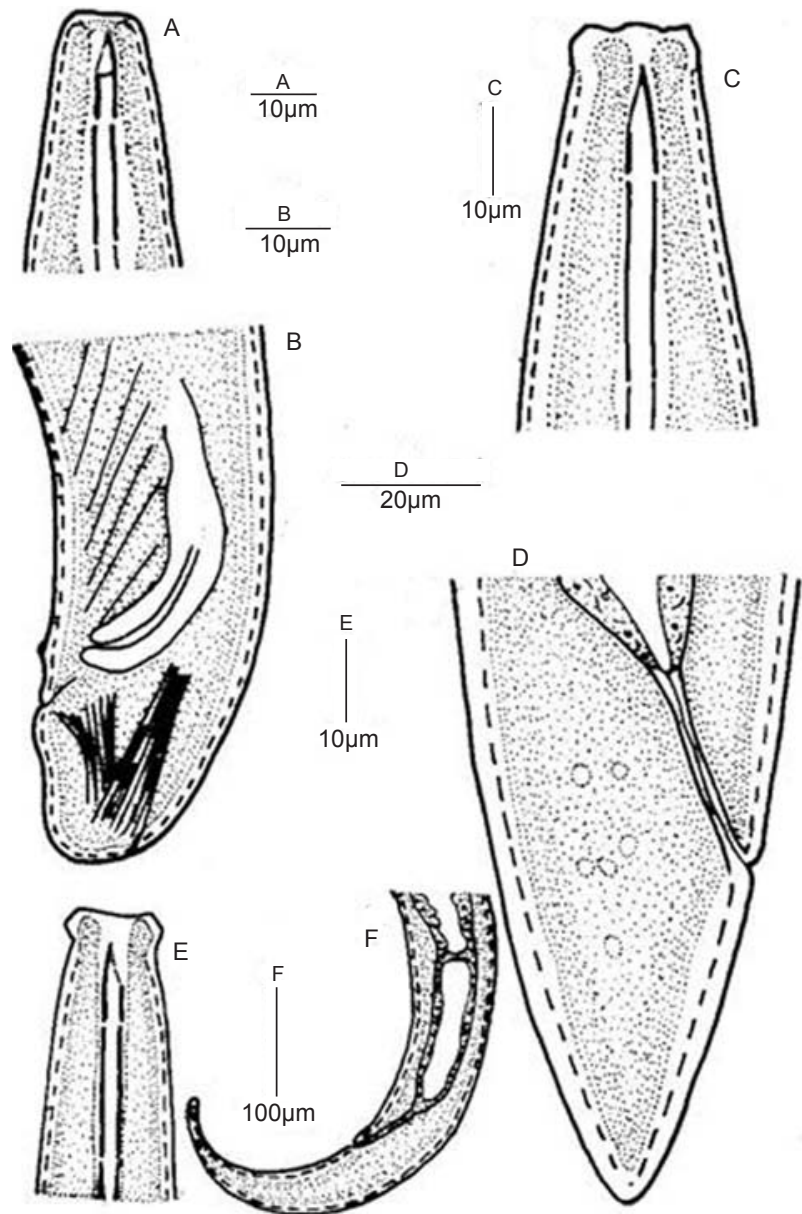

Fig.2. Mesodorylaimus subtiloides (Paetzold, 1958 Andrássy, 1959

A - Anterior end; B - Posterior end.

Aporcelaimellus adoxus Tjepkema, Ferris \& Ferris, 1971

C - Anterior end; D - Posterior end.

Sicorinema sericatum Siddiqi, 1982

$\mathrm{E}$ - Anterior end; F - Posterior end. 

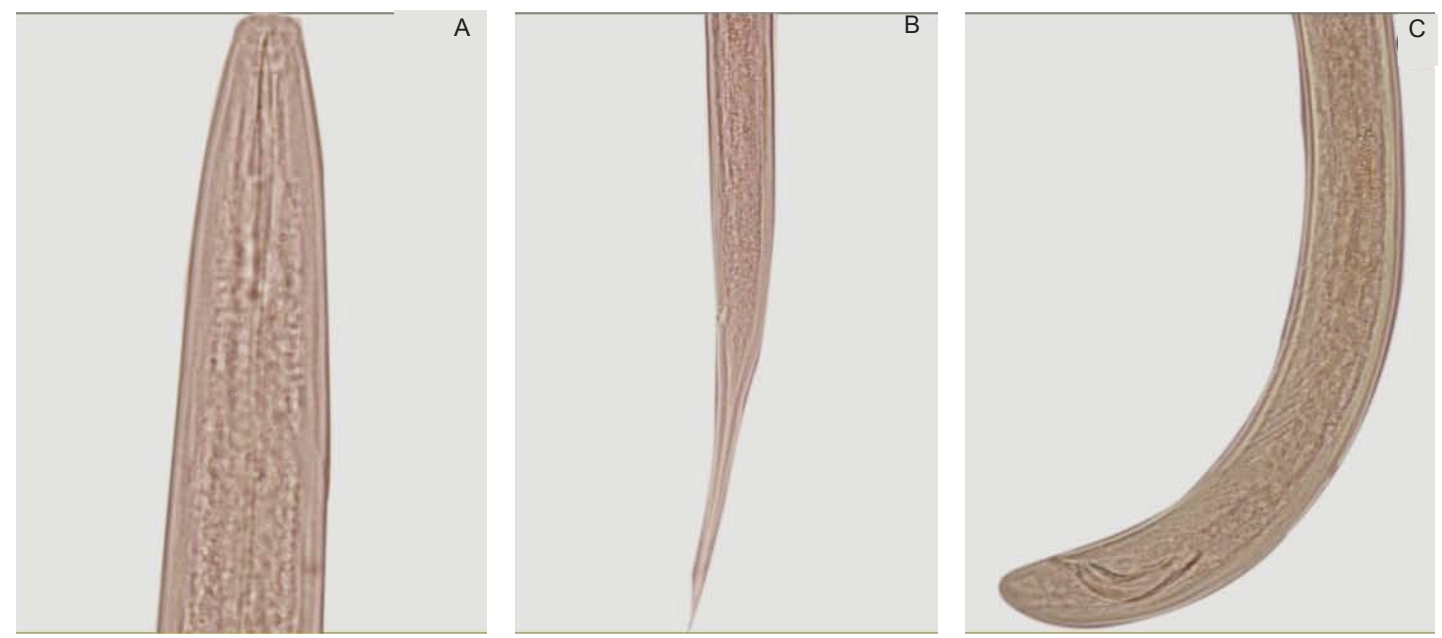

Image 7. Mesodorylaimus subtiloides (Paetzold, 1958) Andrássy, 1959. A - Anterior end; B - Female Posterior end; C - Male Posterior end.
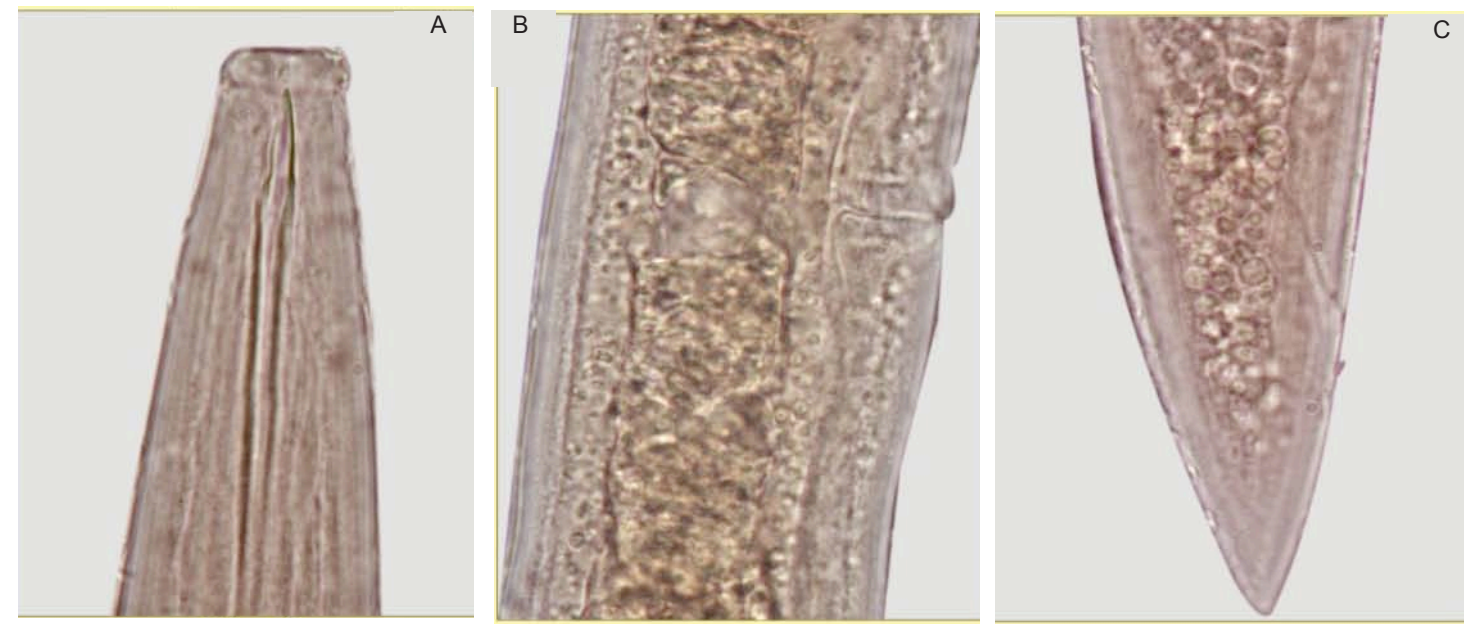

Image 8. Aporcelaimellus adoxus Tjepkema, Ferris \& Ferris, 1971. A - Anteror end; B - Vulval reion; C - Female Posterior region.
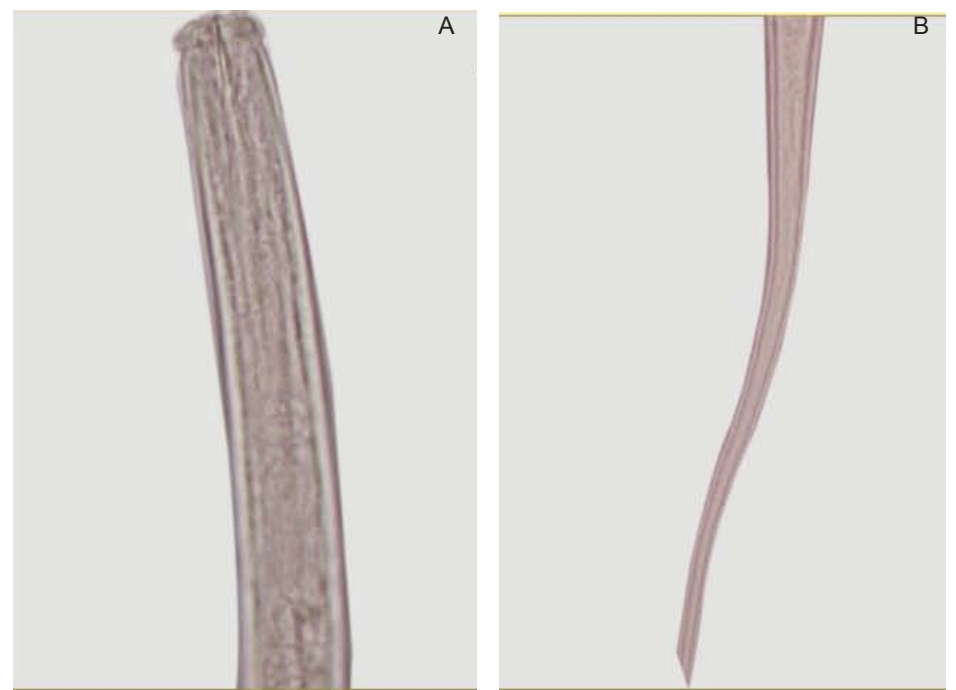

Image 9. Sicorinema sericatum Siddiqi, 1982. A - Anterior region;B - Female Posterior Region. 
the key provided by Andrassy, 1986 for the genus Mesodorylaimus. Measurements of present specimens fit well within the range give by Andrassy, 1986.

\section{Family: Aporcelaimidae Heyns, 1965}

\section{Aporcelaimellus adoxus Tjepkema, Ferris \& Ferris, 1971}

(Fig. 2, C-D and Image $8 \mathrm{~A}-\mathrm{C}$ )

Material examined: Four females, 25.vii.2009, coll. P. Bohra, Reg.No. IV/2235.

Measurements: Females: $\mathrm{L}=1.7-2.2 \mathrm{~mm} ; \mathrm{a}=28$ $33 ; \mathrm{b}=3.4-4.5 ; \mathrm{c}=39-48 ; \mathrm{c}^{\prime}=1.4-1.5 ; \mathrm{V}=45-52$.

Description: Female: Body curved more posteriorly upon fixation. Lips well separated from each other and set off from body by deep constriction. Amphids funnel-shaped, weakly duplex. Odontostyle 14-19 $\mu \mathrm{m}$ long; its aperture $62-65 \%$ of odontostyle length. Odontophore $22-25 \mu \mathrm{m}$ long. Guiding ring plicated anteriorly. Oesophageal expansion gradual, expanded part of oesophagus occupies 55-59\% of total oesophageal length. Cardia conical. Cardiac disc present between oesophagus and intestine. Female reproductive system amphidelphic. Prerectum 60-62 $\mu \mathrm{m}$ long or 1.9-2.1 anal body-width long. Rectum 35$36 \mu \mathrm{m}$ long or 1.0-1.2 anal body-width long. Tail 45 $47 \mu \mathrm{m}$ long or 1.4-1.5 anal body-width long, dorsally convex with blunt tip.

Habitat and locality: Collected from soil around the roots of Rizka (Medicago sativa) at Manda Ki Ghati, Sitamata Wildlife Sanctuary, Chittorgarh, Rajasthan, India.

Remarks: Identification done with original description and measurements provided by Tijpkema, Ferris \& Ferris, 1971.

\section{Family: Qudsianematidae Jairajpuri, 1965 Sicorinema sericatum Siddiqi, 1982} (Fig. 2, E-F and Image 9 A-B)

Material examined: Two females.

Measurements: Females: $\mathrm{L}=0.84-0.89 \mathrm{~mm}$; a $=42-44 ; \mathrm{b}=3.1-3.5 ; \mathrm{c}=14-15 ; \mathrm{c}^{\prime}=3.1-3.9 ; \mathrm{V}=$ 42-44.

Description: Female: Body slender, ventrally arcuate upon fixation. Lip region wider than adjoining body with raised lips and papillae. Odontostyle 10-11 $\mu \mathrm{m}$ long, with $7 \mu \mathrm{m}$ long aperture and wide lumen. Odontophore 17-22 $\mu \mathrm{m}$ long. Expanded part of oesophagous occupies 39-44\% of total oesophageal length. Vulva a small, transverse oval pore-like. Posterior genital branch present (opisthodelphic). Pre-rectum about 30-35 $\mu \mathrm{m}$ or two anal body-width long. Rectum 1.5 anal body width long. Tail 22-25 $\mu \mathrm{m}$ long, ventrally arcuate, regularly tapering to a small rounded terminus.

Habitat and locality: Collected from moist soil around water body at Borunddi maugra, Sitamata Wildlife Sanctuary, Chittorgarh, Rajasthan, India.

Remarks: The identification of species is based on measurements and original description given by Siddiqi, 1982.

\section{Family: Longidoridae Thorne, 1935 \\ Paralongidorus rex (Andrassy, 1986) Hunt, 1993.}

(Fig. 3, A-B and Image $10 \mathrm{~A}-\mathrm{B}$ )

Material examined: Two females, 29.vii.2009, coll. P. Bohra, Reg. No. IV/2239.

Measurements: Females : $\mathrm{L}=2.68-2.74 \mathrm{~mm} ; \mathrm{a}=$ $83-85 ; \mathrm{b}=8.5-8.7 ; \mathrm{c}=90-91 ; \mathrm{c}^{\prime}=0.9-1.0 ; \mathrm{V}=53$.

Description: Female: Long, slender nematodes. Straight to C-shape upon fixation. Lip region continuous with body contour. Amphidial apertures in the form of transverse slits. Amphidial fovea elongate, funnel shaped. Odontostyle long, 55-60 $\mu \mathrm{m}$ long, attenuated and strongly sclerotized. Odontophore 45-50 $\mu \mathrm{m}$ long, without basal flanges. Slender part of oesophagus abruptly expand to form bulboid expanded part which occupies 27-30\% part of total neck length. Vulva transverse. Ovaries paired and reflexed. Tail 28-30 $\mu \mathrm{m}$ long, conoid.

Habitat and locality: Collected from moist soil around Dalia Jalashya on Udaipur to Jaisamand Road, Udaipur District, Rajasthan, India.

Remarks: The measurement of specimens fits well within the range.

Family: Leptonchidae Thorne, 1935

\section{Sclerostylus karri Goseco, Ferris \& Ferris, 1981}

(Fig. 3, C-D and Image $11 \mathrm{~A}-\mathrm{B}$ )

Material examined: Two females, 25.vii.2009; coll. P. Bohra, Reg.No. IV/2237. 

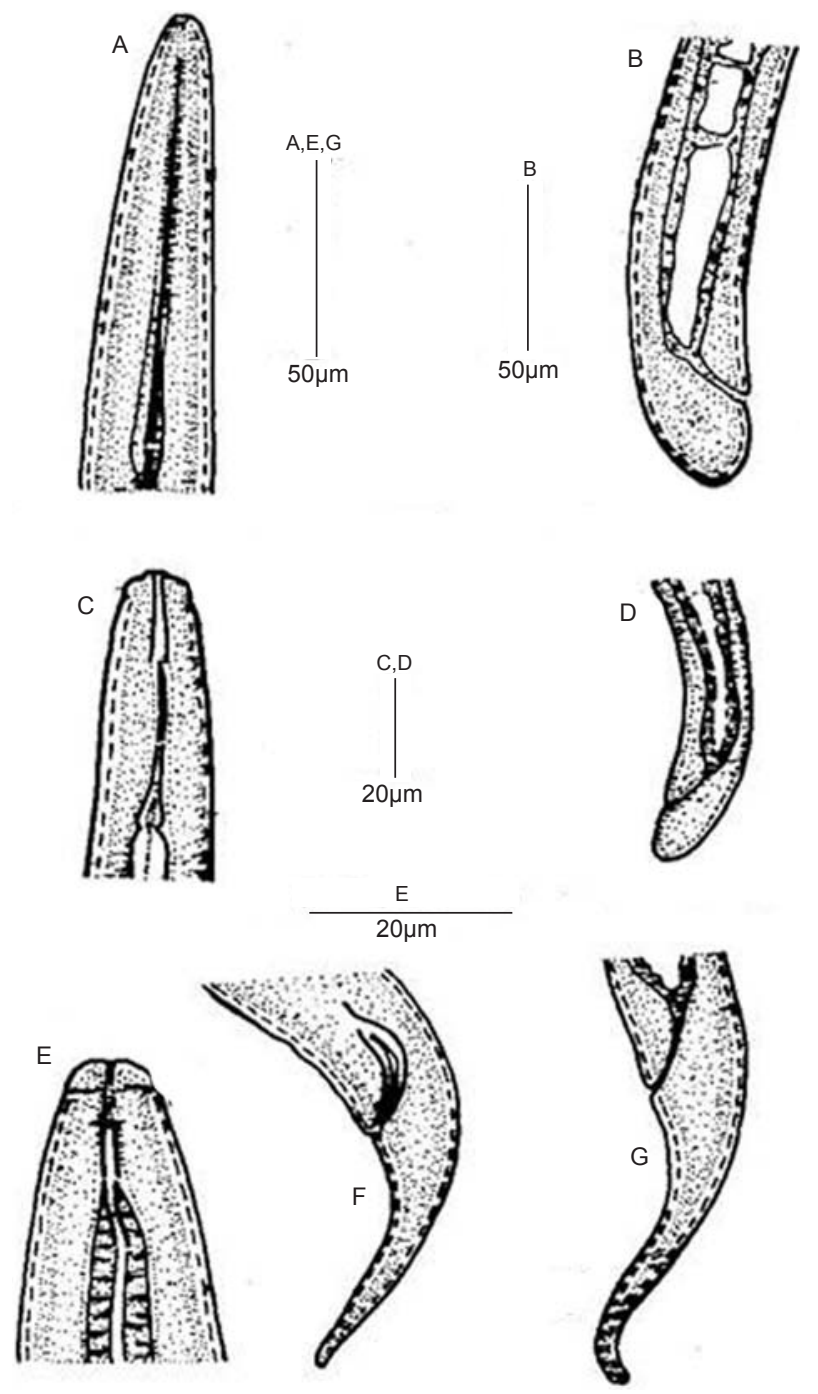

Figure 3. Paralongidorus rex (Andrássy, 1986) Hunt,1993 A - Anterior end; B - Posterior end. Sclerostylus karri Goseco, Ferris \& Ferris, 1971 C - Anterior end; D - Posterior end. Dorylaimoides (L) akon Goseco Ferris \& Ferris, 1976 E - Anterior end; F - Male Posterior end; G - Female posterior end.

Measurements: Females: $\mathrm{L}=0.50-0.72 \mathrm{~mm}$; a $=30-33 ; \mathrm{b}=4.5-4.8 ; \mathrm{c}=48-50 ; \mathrm{c}^{\prime}=1.0-1.1 ; \mathrm{V}=$ 47-48.

Description: Female: Body cylindroids, ventrally arcuate up on fixation. Lip region set off, papillae distinct. Labial disc prominent. Odontostyle 11-13 $\mu \mathrm{m}$ long; odontophore 15-17 $\mu \mathrm{m}$ long with distinct basal flanges. Oesophageal bulb pyriform, about 19-21 $\mu \mathrm{m}$ long or $14 \%$ of oesophageal length. Vulva transverse. Anterior uterine branch about one anal body width long. Posterior gonad normal. Intestine prerectum junction surrounded by three distinct cells, prerectum about $10-12$ anal body width long. Tail 13-15 $\mu \mathrm{m}$ long, about one anal body-width.

Habitat and locality: Collected from soil around roots of unidentified grasses at Borundimaugra, Sitamata Wildlife Sanctuary, Chittorgarh, Rajasthan, India.

Remarks: Measurements of specimens fit well within the range of measurements provided by Goseco, Ferris \& Ferris, 1981.

Family: Mydonomidae Thorne, 1964

\section{Dorylaimoides (Longidorylaimoides) akon Goseco Ferris \& Ferris, 1976 \\ (Fig. 3, E-F and Image 12, A-C)}

Material examined: One female, one male, 29.ix.2009, coll. P. Kadela, Reg. No. 2334.

Measurements: Female: $\mathrm{L}=1.36 \mathrm{~mm} ; \mathrm{a}=34.05 ; \mathrm{b}$ $=6.8 ; \mathrm{c}=12.16 ; \mathrm{c}^{\prime}=7.4 ; \mathrm{V}=44 ;$ Male: $\mathrm{L}=1.28 \mathrm{~mm}$; $\mathrm{a}=40.25 ; \mathrm{b}=5.8 ; \mathrm{c}=19.8 ; \mathrm{c}^{\prime}=3.10, \mathrm{~T}=63-65$.

Description: Female: Body cylindroids, ventrally arcuate upon fixation. Lip region slightly off set, outer liplets and papillae distinct. Odontostyle $12 \mu \mathrm{m}$ long; ventral arm $4 \mu \mathrm{m}$ long. Odontophore $13 \mu \mathrm{m}$ long, arcuate. Oesophageal bulb cylindroids which occupies $50 \%$ of oesophageal length. Vulva transverse, ovaries paired. Prerectum distinct. Rectum $20 \mu \mathrm{m}$ long or one anal body diameter. Tail $112 \mu \mathrm{m}$ long, elongate conoid or 7.4 anal body width, terminus dorsally bent. Male: Spicules $40 \mu \mathrm{m}$ long. Lateral guiding pieces present. Series of 5-8 ventromedian supplements plus adanal pair. Tail elongate conoid 3.10 anal body-width long.

Habitat and locality: Collected from moist soil around the bank of Fatehsagar Lake, Udaipur District, Rajasthan, India.

Remarks: Measurements fit well within the range given by Goseco, Ferris \& Ferris, 1976.

\section{REFERENCES}

Andrássy, I. (1986). The genus Mesodorylaimus Andrassy, 1959 and its relatives (Nematoda: Dorylaimidae). Acta Zoologica Hungarica 32(3-4): 207-261.

Andrássy, I. (1986). Paralongidorus rex sp.n., a new nematode species from Hungary. Allattani Kozlemenyek 73: 115118.

Arya, H.C. (1957). Root-knot disease of tomatoes in Jodhpur. Science \& Culture 22: 391-392. 

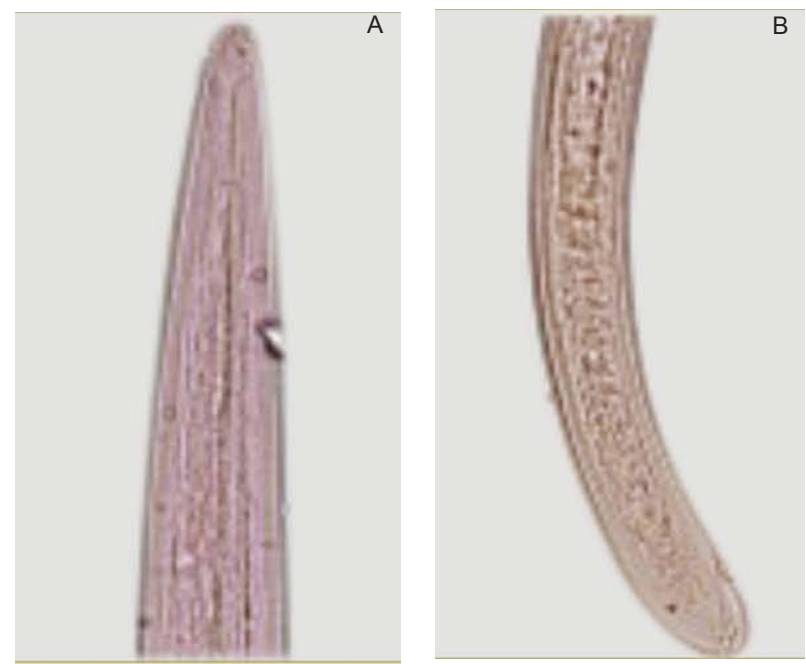

Image 10. Paralongidorus rex (Andrássy, 1986) Hunt,1993. A - Anterior region; B - Posterior end;
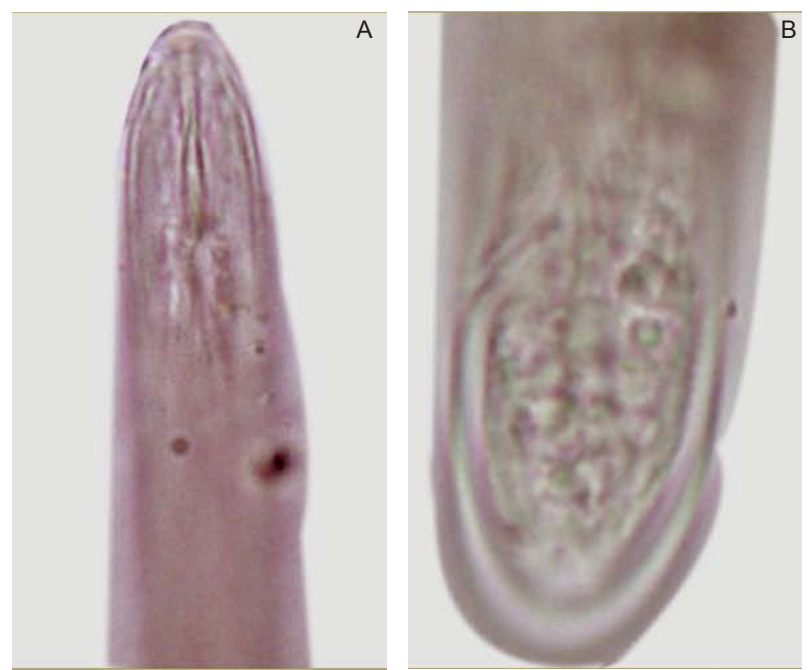

Image 11. Sclerostylus kari Goseco, Ferris \& Ferris,1971. A Anterior region; B - Posterior.region.
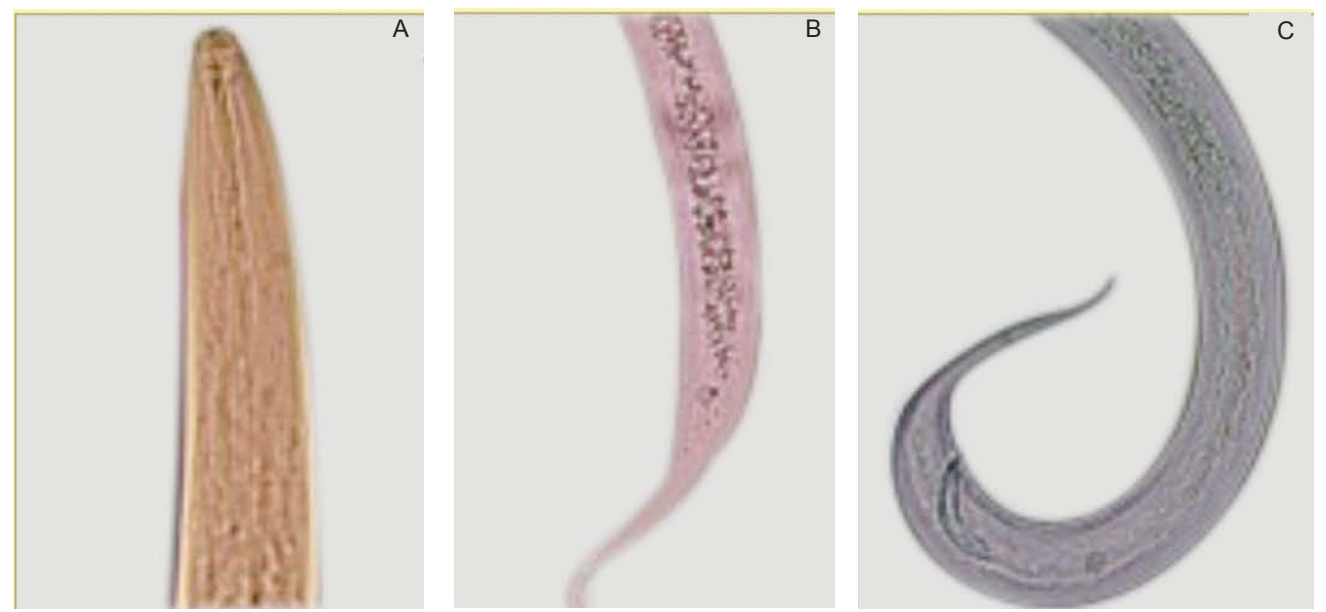

Image 12. Dorylaimodes(L)akon Goseco, Ferris \& Ferris,1976. A - Anterior end; B - Male Posterior end; C - Female Posterior end.

Bajaj, H.K. \& M.S. Jairajpuri (1979). A review of the genus Xiphinema Cobb, 1913 with descriptions of species from India. Records of Zoological Survey India 75: 255-325.

Baqri, Q.H. \& P. Bohra (2001). Nematodes from Rajasthan, India. I. Six new species of Dorylaimida. Nematology 3(2): $113-127$.

Baqri, Q.H.\& P. Bohra (2003). Prothornenema gen.n. and four new species of Dorylaimoidea (Nematoda: Dorylaimida) from India. International Journal of Nematology 13(2): 185-194.

Bohra, P. (2008). Qualitative and Quantitative Studies of Plant and Soil Nematodes associated with crops of economic importance in Rajasthan. Records Zoological Survey India Occasional Paper 278: 1-180.

Bohra, P. (2011). Pictorial Hand Book on Plant and Soil Nematodes of Rajasthan. Published by Director of Zoological Survey of India, Kolkata, 243pp+31pls.
Bohra, P. \& Q.H. Baqri (2000). Nematodes from Rajasthan II. Six new records of order Dorylaimida from India. Records Zoological Survey India Kolkata 98(4): 117-122.

Bohra, P. \& Q.H. Baqri (2003). Nematodes from Rajasthan, India. IV. Seventeen species as new record from the State. Records Zoological Survey India Kolkata 101(3\&4): 129145.

Goseco, C.G., V.R. Ferris \& J.M. Ferris (1976). Revision in Leptonchoidea (Nematoda: Dorylaimida). Dorylaimoides in Dorylaimoididae, Dorylaimoidinae; Calolaimus and Timmus n. gen. in Dorylaimoididae, Calolaiminae; and Miranema in Miranematidae. research bulletin No. 941: 1-45. Purdue Univrsity, Agricultural Experiment Station, West Lafoyette, Indiana, 47907.

Goseco, C.G., V.R. Ferris \& J.M. Ferris (1981). Sclerostylus n. gen. from Panama and other Neotropical species of Leptonchoidea (Dorylaimida). Journal of Nematology 
13(1): 79-86.

Hunt, D.J. 1993. Aphelenchida, Longidoridae and Trichodoridae: Their Systematic and Bionomics. CAB International. Research Bulletein No. 941: 1-45. Purdue University, Agricultural Experiment Station, West Lafoyette, Indiana, 47907, xi-339pp.

Jairapuri, M.S. \& D.J. Hunt (1984). The taxonomy of Tylenchorhynchinae(Nematoda: Tylenchida)with longitudinal lines and ridges. Systematic Parasitology 15: 261-268.

Jairajpuri, M.S. \& W. Ahmad (1992). Dorylaimida - Free living, Predaceous and Plant-parasitic Nematodes. Oxford \& IBH Publishing Co. Pvt. Ltd., New Delhi, v+449pp.

Khera, S. (1967). Acrobelinema cornis n. g., n. sp. subfamily Acrobelinae Thorne from rhizosphere of millets from India. Indian Journal of Helminthology 21(2): 159-163.

Khera, S. (1969). Nematodes from the banks of still and running waters. VI. Order Rhabditida from Sewar. Journal of Helminthology XLIII (3\&4): 347-363.

Khera, S. (1971). Nematodes from the banks of still and running waters. VII. Family Monhysteridae. Nematologica (1970) 16: 492-502.

Nandkumar, C. \& S. Khera (1969). Paurodontus aberrans n. sp. (Nematoda: Tylenchida) with a note on a special branch of the oviduct. Indian Journal of Helminthology 21: 1-15.

Nandkumar, C. \& S. Khera (1970). A new nematode species Pratylenchus mulchandi from Millets of Rajasthan, Indian Journal of Phytopathology 22: 359-363.

Sethi, C.L. \& G. Swarup (1968a). Plant parasitic nematodes of north-western India. III. The genus Pratylenchus: Indian Journal of Phytopathology 24: 410-412.

Sethi, C.L. \& G. Swarup (1968b). Plant parasitic nematodes of north-western India. I. The genus Tylenchorhynchus. Nematologica 14: 77-88

Sher, 1966. Revision of the Hoplomaiminae (Nematoda) VI. Helicotylenchus Steiner, 1945. Nematologica 12: 1-56.

Siddiqi, M.R. (1972). On the genus Helicotylenchus Steiner, 1945 (Nematoda: Tylenchida), with description of nine new species. Nematologica 18: 74-91.

Siddiqi, M.R. (1979). The origin and phylogeny of nematodes of order Tylenchida. Nematropica 9: 109.

Siddiqi, M.R. (1982). Sicorinema gen.n. and Moshajia gen.n. (Dorylaimida:Crateronematidae) with description of four new species. Nematologia Mediterranea 10: 157-166.

Tikyani, M.G. \& S. Khera (1968). Neopaurodontus asymmetricus n. g., n. sp. (Nematoda: Paurodontidae) from rhizosphere of great millet. Indian Journal of Helminthology 20: $34-39$.

Tikyani, M.G., S. Khera, \& G.C. Bhatnagar (1969). Helicotylenchus goodi n. sp. (Nematoda: Paurodontidae) from rhizosphere of great millet. Zoologischer Anzeiger 182: 420-423.

Tjepkema, R. Virginia, V.R. Ferris \& J.M. Ferris (1971). Review of the genus Aporcelaimellus Heyns, 1965 and six species group of the genus Eudorylaimus Andrassy, 1959 (Nematoda: Dorylaimida). Research bulletin no. 882: pp: 1-51. Purdue University. Agricultural Experiment Station, Lafoyette, Indiana. 